\title{
Stepping Up Glaucoma Management: When and How?
}

\author{
Ivan Goldberg ${ }^{*}, 1,2,3$ \\ ${ }^{I}$ Department of Ophthalmology, University of Sydney, Australia \\ ${ }^{2}$ Glaucoma Unit, Sydney Eye Hospital, Australia \\ ${ }^{3}$ Eye Associates, Australia
}

\begin{abstract}
Glaucoma management aims to preserve patients' quality of life by halting progressive visual loss. Intraocular pressure reduction is the pivotal strategy: we establish a "target" pressure based on extent of visual damage, threat to visual performance, risk profile for further damage, pre-treatment pressure levels, family history and a patient's life expectancy. Once an acceptable treatment regimen has been instituted, review ascertains whether damage has advanced and/or the risk of progressive damage has changed. Increased damage and/or increased risk of progressive damage suggest accelerated treatment, and lowered target pressures.

When should management be stepped up? When a glaucoma patient, or a glaucoma suspect is getting worse and it matters, and when an individual is at increased risk of getting worse and it matters.

At each review, the ophthalmologist seeks change in disease status: is it stable, uncertain whether or not it is stable, or progressing? Equally, the level of risk of further damage is assessed: is it unchanged, uncertain whether it is unchanged, or increased? If both disease status and risk are stable, the patient continues as is; if either or both have changed, treatment either can be initiated for suspects, or accelerated for patients. This means, intervene to reduce the perceived risk of further damage, and this means reducing or further reducing pressure.
\end{abstract}

Management of glaucoma is an ongoing challenge: the disease is incurable. It is usually progressive and the damage it causes is irreversible. In its early stages, symptoms are vague unless sought specifically [1], and presentation is often relatively late in the disease course [2]. The ophthalmologist's goal is to maintain the patient's Quality of Life. This is achieved by halting or at least slowing significantly further visual damage. Besides ensuring the patient's general health status is optimal, intraocular pressure (IOP) reduction is the pivotal strategy - this is the only proven risk reduction treatment.

Inherent in this approach is the establishment of a "target" IOP level, guessed to be likely to ensure the patient's visual security from further glaucomatous damage. Many factors are taken into account in setting a target IOP for a particular patient [3], including extent of visual damage already suffered, the threat of that damage to visual performance (such as proximity to fixation), the risk profile for further damage (such as presence of pseudoexfoliation syndrome), the pre-treatment IOP level, family history and the patient's estimated life expectancy. Lowering IOP is not an end in itself, but a strategy to protect the individual patient from the threat of glaucoma-induced visual disability.

These same principles apply to patients who are "glaucoma suspects": perceived to be at increased risk to develop glaucomatous visual damage but with no definitive signs of structural or functional damage at the time of

*Address correspondence to this author at the Department of Ophthalmology, University of Sydney, Australia; E-mail: eyegoldberg@gmail.com assessment. These include patients with ocular hypertension, glaucoma-like optic discs, and a family history of glaucoma, especially of blinding glaucoma.

Following 'institution' of an acceptable treatment regimen, the challenge is to review appropriately so as to ascertain whether or not further damage has occurred, and as to what is the 'ongoing risk status' for further damage. Management may need to be accelerated ("stepped up") when the ophthalmologist identifies further damage or a change in the risk profile. Accelerating treatment means increasing efforts at IOP reduction, and/or resetting the target pressure. Therefore, these principles apply to glaucoma suspects being monitored to answer the question: does treatment need to be initiated?

When should management be stepped up? When a glaucoma patient or a glaucoma suspect is getting worse and it matters, and when such an individual is at increased risk of getting worse and it matters.

At each review, the ophthalmologist assesses any change in disease status: is it stable, uncertain whether or not it is stable, or progressing? Equally, the level of risk of further damage is assessed: is it unchanged, uncertain whether or not it is unchanged, or increased? (Table 1). If both disease status and risk are assessed as stable, the patient can continue as is; if either or both have changed, treatment either can be initiated for suspects, or "stepped up" for patients. This means, actively intervening to reduce the perceived risk of further damage, and this means reducing or further reducing IOP. 
Table 1 Decision Square for Management of Glaucoma and Suspects [4]

\begin{tabular}{l|c|c|c|c|}
\hline \multirow{2}{*}{ Risk } & \multicolumn{3}{|c|}{ Disease Status } \\
\cline { 2 - 4 } & Stable & Uncertain & Progressing \\
\hline \hline Increased & + & ++ & +++ \\
\hline Uncertain & Reassess risk & Reassess risk \& disease status & ++ \\
\hline Unchanged & - & Reassess disease status & + \\
\hline+ & $\begin{array}{l}\text { No change needed. } \\
++\end{array}$ \\
++ & \multicolumn{3}{|c|}{$\begin{array}{l}\text { Modest acceleration of management. } \\
\text { Aggressive acceleration of management. }\end{array}$} \\
\hline
\end{tabular}

What are the risk factors? For onset of glaucomatous damage, they include older age, strong family history, higher IOP, glaucomatous damage in the contralateral eye, and lower central corneal thickness, pigment dispersion and pseudo-exfoliation syndromes as well as disc or disc margin haemorrhage, large cup-to-disc ratio (in the context of disc size), diabetes, and vasospastic syndromes like migraine and Raynaud's phenomenon. For glaucoma progression, add to these lower ocular perfusion pressure (lower blood pressure) and more advanced damage at baseline (such as damage in both visual hemifields).

To tailor therapy appropriately for individual patients, it is helpful to consider risk categories [4]:

1. Glaucoma with a high 5-year risk for progression, or a high 5-year risk of visual disability, with a target IOP reduction of $40 \%$ or more from baseline [5-7], or 1-2 standard deviations below the patient's ethnic population mean (whichever is the lower), if the therapeutic index (potential benefit assessed against possible harm) justifies it.

- Moderate to advanced glaucoma damage with correlating structural and functional loss.

- Demonstrated progression - especially if over a short time.

- Higher IOP.

- Bilateral visual field loss.

- Field loss involving both superior and inferior hemifields.

- Field loss threatening fixation.

- Pigmentary and/or pseudo-exfoliation syndromes or other secondary glaucomas.

- Quality of life already affected by damage.

- Younger age patient with advanced damage.

- Angle-closure glaucoma.

2. Glaucoma with moderate 5-year risk for progression [8], or glaucoma suspect with high risk for conversion to glaucoma [9], with a target IOP reduction of $30 \%$ or more from baseline, or the population mean (whichever is the lower).
- Mild glaucomatous damage with correlating structural and functional loss and higher baseline IOP.

- Mild to moderate glaucomatous damage with lower baseline IOP.

- Primary angle-closure with peripheral anterior synechiae and high IOP.

- Younger age with damage.

3. Glaucoma suspect at moderate 5-year risk for damage, in whom "masterful inactivity" with monitoring for change is an option, as is a modest IOP reduction, depending on patient preferences and overall risk. Treat if risks increase (or damage identified) with a target IOP reduction of $20 \%$ or more from baseline, or 1 standard deviation above the population mean, whichever is the lower. The fellow eye might require the same target IOP depending on risk status.

- Fellow of eye with established damage, excluding unilateral secondary glaucoma.

- Ocular hypertension with multiple additional risk factors (thin central corneal thickness, higher IOP, glaucoma-like disc).

- Recurrent disc haemorrhages.

- Pseudo-exfoliation syndrome.

- Younger age.

4. Glaucoma suspect at lower risk for visual loss [10], in whom careful establishment of structural and functional baselines and appropriate review frequency will allow "masterly" monitoring.

- Ocular hypertension.

- Older age.

- Family history.

- Primary angle-closure suspect.

- $\quad$ Pigment dispersion syndrome with normal IOP.

- Glaucoma-like disc.

- A combination of risk factors of lesser importance including steroid responder, myopia, diabetes mellitus, systemic hypertension, uveitis.

What this implies is recognition of the parallel static and dynamic profiles we create and modify for each patient: static is the baseline assessment of any damage that exists. Dynamic is ongoing assessment of damage that exists (structural and/or functional) and the risk either of development (glaucoma suspects) or progression (glaucoma patients) of such damage.

Following dynamic re-assessment of a patient, the ophthalmologist has three choices. If status and risk are stable, offer reassurance and determine appropriate timing for the next review. If risk profile has changed (e.g. increase in IOP), opt for a "soft push": trial an increase in therapy by switching or increasing medications or by offering laser trabeculoplasty. If damage and especially the risk of visual disability have increased, pursue a "hard push": increase 
treatment aggressively by adding medications and/or performing laser trabeculoplasty and/or considering surgery. Review needs to ensure that the increased measures have achieved their goals: reduction in risk of further damage and stabilization of damage.

An approach like this allows us as clinicians to determine a personalized therapeutic index: potential benefit of intervention for that patient, versus the possibility of causing harm. It allows us to involve the patient actively and meaningfully in his/her decision-making process at every step along the way. For a chronic, incurable, usually progressive disease causing irreversible damage, often asymptomatically, this is critical for management success in the longer-term.

\section{REFERENCES}

[1] Goldberg I, Clement CI, Chiang TH, et al. Assessing quality of life in patients with glaucoma using the glaucoma quality of life-15 (GQL-15) questionnaire. J Glaucoma 2009; 18: 6-12.

[2] Deva NC, Insull E, Gamble GD, Danesh-Meyer HV: Risk factors for first presentation of glaucoma with significant visual field loss. Clin Exp Ophthalmol 2008; 36(3): 217-21.

[3] European Glaucoma Society: Terminology and Guidelines for Glaucoma: Flowcharts VI: Target IOP. $3^{\text {rd }}$ ed. Savona: Editrice Dogma 2008.
[4] South East Asia Glaucoma Interest Group: Asia Pacific Glaucoma Guidelines: Assessment: Risk Categories and Treatment Targets. $2^{\text {nd }}$ ed. Hong Kong: Scientific Communications International 2008 [Available at www.seagig.org].

[5] Leske MC, Heijl A, Hussein M, et al. Early Manifest Glaucoma Trial Group: Factors for glaucoma progression and the effect of treatment: the early manifest glaucoma trial. Arch Ophthalmol 2003; 121: 48-56.

[6] Collaborative Normal-Tension Glaucoma Intervention Study Group: The effectiveness of intraocular pressure reduction in the treatment of normal-tension glaucoma. Am J Ophthalmol 1998; 126: 498-505.

[7] The AGIS Investigators. The Advanced Glaucoma Intervention Study (AGIS): 7. The relationship between control of intraocular pressure and visual field deterioration. Am J Ophthalmol 2000; 130: 429-40.

[8] Heijl A, Leske C, Bengtsson B, et al. Early manifest glaucoma trial group: reduction of intraocular pressure and glaucoma progression: results from the early manifest glaucoma trial. Arch Ophthalmol 2002; 120: 1268-79.

[9] Kass MA, Heuer DK, Higginbotham EJ, et al. The ocular hypertension treatment study: a randomized trial determines that topical ocular hypotensive medications delays or prevents the onset of primary open-angle glaucoma. Arch Ophthalmol 2002; 120: 701-13.

[10] Gordon MO, Beiser JA, Brandt JD, et al. The ocular hypertension treatment study: baseline factors that predict the onset of primary open-angle glaucoma. Arch Ophthalmol 2002; 120: 714. 